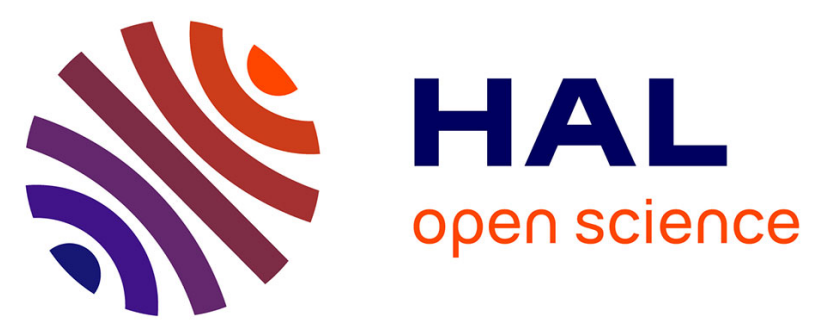

\title{
Ultrasonic welding of thermoplastic composites: a numerical analysis at the mesoscopic scale relating processing parameters, flow of polymer and quality of adhesion
}

Arthur Lévy, Steven Le Corre, Arnaud Poitou

\section{To cite this version:}

Arthur Lévy, Steven Le Corre, Arnaud Poitou. Ultrasonic welding of thermoplastic composites: a numerical analysis at the mesoscopic scale relating processing parameters, flow of polymer and quality of adhesion. International Journal of Material Forming, 2014, 7 (1), pp.39-51. 10.1007/s12289-0121107-6 . hal-03191184

\author{
HAL Id: hal-03191184 \\ https://hal.science/hal-03191184
}

Submitted on 8 Apr 2021

HAL is a multi-disciplinary open access archive for the deposit and dissemination of scientific research documents, whether they are published or not. The documents may come from teaching and research institutions in France or abroad, or from public or private research centers.
L'archive ouverte pluridisciplinaire $\mathbf{H A L}$, est destinée au dépôt et à la diffusion de documents scientifiques de niveau recherche, publiés ou non, émanant des établissements d'enseignement et de recherche français ou étrangers, des laboratoires publics ou privés. 


\title{
Ultrasonic welding of thermoplastic composites: a numerical analysis at the mesoscopic scale relating processing parameters, flow of polymer and quality of adhesion
}

\author{
Arthur Levy • Steven Le Corre • Arnaud \\ Poitou
}

Received: May 11, 2012

\begin{abstract}
Ultrasonic continuous welding of thermoplastic composite plates is a very promising process of particular interest for the assembly of aeronautics large parts. Its modeling and simulation however suffers from the difficulty of accounting for the very different time scales that rule the thermo-mechanical phenomena at the level of the adhesion zone. This problem was addressed in our previous works and led to an original simulation tool presented in (Levy et al [13], 2011, Eur. J. Mech. A/Solids, 30(4)). In this paper, the adopted time-homogenized multiphysical modeling of the flow at the mesoscopic scale of the energy directors is first presented. Then, using the numerical software in a $2 \mathrm{D}$ approach, an extensive numerical parametric study of the process is presented. The phenomena allowing welding are confirmed to be an initial strain concentration in the energy director, and the formation of a flowing fold. The influence of the following process parameters are finally investigated: amplitude of vibrations, holding force of the sonotrode, thickness of the plates, radius of curvature at the tip of the director, angle of the director. Process efficiency and weld quality is evaluated through simple indicators such as the equivalent stiffness analysis, the healing degree and the risk of porosity entrapment. The present study, carried at the mesoscopic scale, provides a better understand-
\end{abstract}

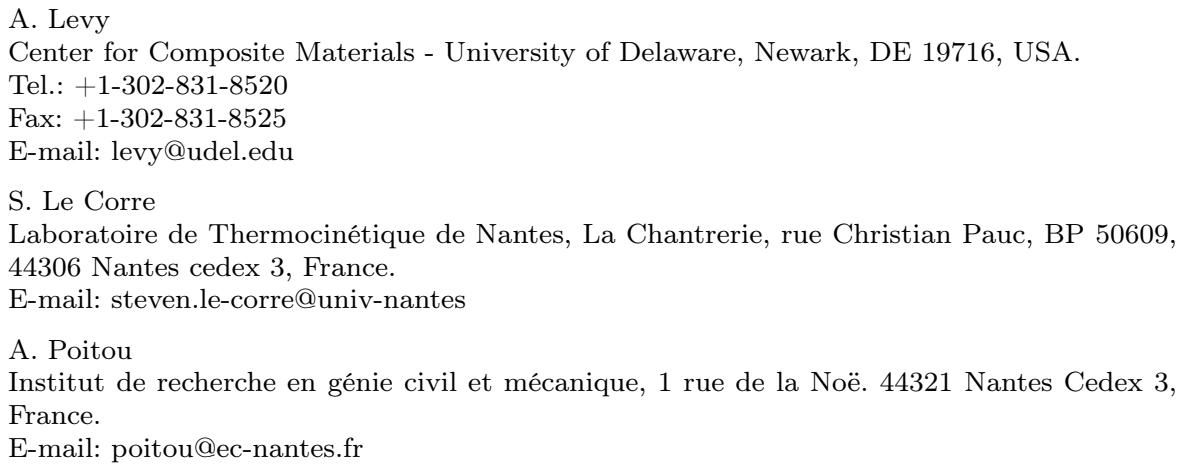


ing of the complex interactions between physical and process parameters and enable to draw important technological conclusions for the design of energy directors.

Keywords ultrasonic welding - multiphysical modeling $\cdot$ numerical analysis · healing $\cdot$ process parameters influence $\cdot$ geometry effects

\section{Introduction}

Thanks to their good specific properties, composite materials tend to replace traditional materials such as metallic materials in the aeronautic industry. Whereas thermoset matrix composite were widely used in this industry for several years, thermoplastic matrix composites nowadays tend to replace them. Besides the good physico-chemical properties of the thermoplastic matrix such as PolyEtherEtherKetone (PEEK), the ability to melt the matrix opens perspectives to a variety of new and possibly more efficient forming processes. Contrary to thermoset composites, processes involving thermoplastic matrices do not require the curing step, which can lead to two main improvements: (i) much shorter cycle times and (ii) no need for a confinement or autoclave step. Huge parts, which could not fit in any reasonable size stove may therefore be manufactured without limit.

Of particular interest is also the welding possibility provided by thermoplastic composites. Thanks to the ability of the matrix to melt, two parts can be assembled thanks to local heating and melting of the interface. The difficulty in welding organic composite then comes from their low thermal conductivity which therefore oriented technological investigations towards the idea of local heating of the interface between the two parts to be assembled. At this day, different technologies were considered such as resistance welding [21] or induction welding [3]. In those processes a metallic grid is needed and remains trapped at the interface after welding, which induces lower interface properties.

This work focuses on the promising ultrasonic welding process, which does not suffer from such a drawback and may lead to more efficient assemblies. In this process, in order to achieve the local heating, triangular bulges, called energy directors, are molded on one of the plate to be welded. The assembly is then positioned under a tool called sonotrode which applies simultaneously a constant load and a harmonic ultrasonic compression (Fig. 1(a)). It is now commonly known that the strain, located in the director, induces heating because of viscous dissipation [24], [4], [18]. The director then melts and flows at the interface to allow welding (Fig. 1(b)).

First experiments were performed by EADS IW with the so-called "continuous process", patented in 2007 by Soccard [19], where the sonotrode moves along the energy director direction. First results reveal a good mechanical quality of the welding. In particular the advance of the sonotrode enables air removal along the director and avoids the trapping of bubbles. Moreover, it would allow one to assemble large parts using continuous weld lines, where 


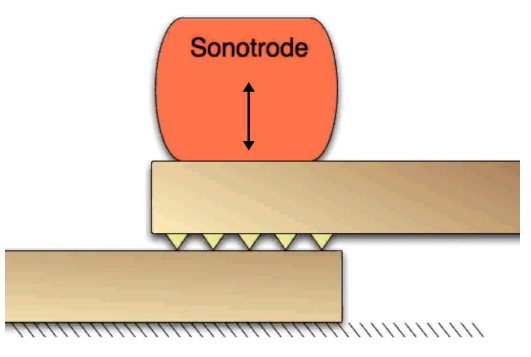

(a) Assembly.

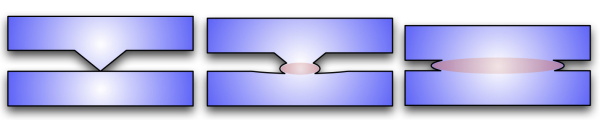

(b) Flow at the interface.

Fig. 1: Principle of the ultrasonic welding technique.

the "static" process (without sonotrode advance) limits the welding area to the size of the sonotrode. This opens possibilities for this process to be used at an industrial level to assemble large parts keeping an excellent weld quality.

The main phenomena occurring in this process, initially outlined by Benatar and Gutowski [4] can be summarized as: (i) mechanics and vibration of the parts, (ii) viscoelastic heating, (iii) heat transfer, (iv) flow and wetting, and $(v)$ intermolecular diffusion. Using a time homogenization technique based on the use of double time-scale asymptotic expansions, a simultaneous modeling of the vibration, heat transfer and flow was proposed in a previous work [14]. This theoretical analysis showed that thanks to the good time scales separation implied by such a process, the heating and flow of the energy directors could be modeled by three problems, two macro-temporal ones, at the time scale of the squeezing, that enable to describe the average heating and flow of the polymer, and a micro-temporal one, at the time scale of one period of vibration, that enable to compute the self-heating of the viscoelastic material. A specific numerical tool was then developed in order to simulate the flow of polymer at the interface [13]. This original finite element software, based on the use of an Eulerian framework associated with level-set functions to describe the evolution of the polymer outer surface, was validated and proved to be able to describe the main physical phenomena of such a complex industrial process.

In this paper, basing on the use this simulation tool, we investigate the influence of various process parameters on the quality of the welding and bring new elements for a better understanding and optimization of this technology. In section 2, we remind the modeling and simulation tools and introduce two criteria that can qualify a good welding, in order to compare the efficiency of different technical solutions. In section 3, a reference simulation, with realistic material parameters typical of PEEK polymer composites, is first detailed. It enables to analyze the two coupled phenomena at the origin of welding: heating and flow. As a major objective of this paper, the influences of five parameters of high interest for the industrial manufacturer are then investigated: the two main processing parameters, (i) amplitude of vibration and (ii) holding force of 


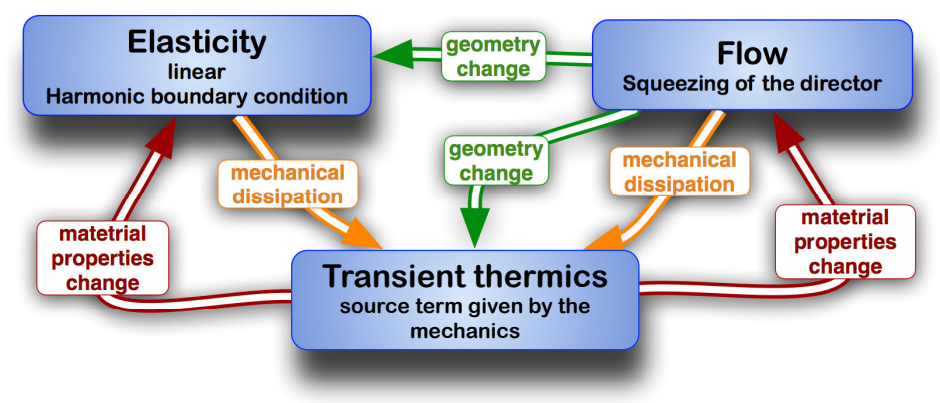

Fig. 2: Modeling of the thermomechanical problem using three coupled boundary value problems.

the sonotrode, (iii) the effect of plates thickness that shows the technological limitations of such a process, and the influence of the director's geometry through the variation of (iv) the smoothness of the tip and $(v)$ the sharpness of triangles.

\section{Methods}

By opposition to the studies at the macroscopic scale (see for example Ha Minh [9]), where one is interested in phenomena such as vibrations, dimension tolerance or residual stresses effects, this work focuses on the so-called mesoscopic scale. In this approach, which has been the subject of previously cited works $[24],[4],[18]$, the objective is to understand the effect of process parameters on the welding mechanisms and the quality of the adhesion at the interface. One therefore has to consider the heating and flow of the energy directors at the interface between the two composite plates, which height are about $300 \mu \mathrm{m}$. The mesoscopic scale discussed in the following is defined such that it has a characteristic dimension of $100 \mu \mathrm{m}$.

\subsection{Modeling and simulation of the process}

\subsubsection{Multiphysical modeling}

This rather classical thermo-mechanical problem including self-heating of a polymer presents a strong difficulty due the huge difference between the welding time $(\sim 1 \mathrm{~s})$ and the vibration period $\left(\sim 10^{-5} \mathrm{~s}\right)$ which makes a full direct simulation of the problem unaffordable. This problem was addressed in our previous work [14], basing on a time-homogenization technique which leads to an original model consisting of three coupled physics as illustrated in Fig. 2. 
Problem 1: An elasto-statics problem at the short time scale describes the effect of the vibration induced by the sonotrode. The Young's modulus depends on temperature according to the following law, which was fitted from experimental data on PEEK provided by several authors [4], [8], [15]:

$$
E(\theta)=2,810^{9}\left[0.5-\frac{\arctan \left(\frac{\theta\left[^{\circ} C\right]-140}{20}\right)}{\pi}\right]+1,610^{8} \mathrm{~Pa} .
$$

Problem 2: The heating at long time scale is described by a usual conduction convection problem where a self heating source term $Q$ due to the vibration is added and computed from problem 1 as:

$$
Q=\frac{\omega E^{\prime \prime}}{2}\langle\varepsilon: \varepsilon\rangle
$$

where $\omega$ is the pulsation of the sonotrode, $E^{\prime \prime}$ is the loss modulus of the polymer, and $\varepsilon$ is the elastic strain [24], [4], [25], [22], [14]. In this equation, $\langle\cdot\rangle$ operator represents the average over one time-period of vibration.

Problem 3: A Stokes problem then describes the flow of polymer. In order to account for the shear thinning and large strain effects in the polymer, a Carreau type law is adopted for the viscosity evolution:

$$
\eta=\eta_{0}(T)\left(1+\left(\lambda_{c} D_{e q}\right)^{2}\right)^{\frac{m-1}{2}},
$$

where $\eta_{0}(T)$ denotes the Newtonian viscosity at a given temperature $T, \lambda_{c}$ the Carreau characteristic time, $m$ the Carreau index and $D_{e q}=\sqrt{2 \mathrm{D}: \mathrm{D}}$ the equivalent strain rate, $D$ being the strain rate tensor. The Newtonian viscosity is furthermore assumed to depend on temperature according to an Arrhenius law:

$$
\eta_{0}(T)=A \exp \left(\frac{E_{a}}{R T[K]}\right)
$$

where $A$ is a material parameter, $E_{a}$ is the activation energy of the material and $R=8.31 \mathrm{JK}^{-1} \mathrm{~mol}^{-1}$ is the gas constant. These choices where directly inspired by results of the literature on the rheology of PEEK [17] and used values are given in table 1 .

\subsubsection{Numerical tool}

In order to solve the three boundary value problems on the initially triangular shape of the director, a specific numerical tool was developed [13]. It enables to handle both the multiphysical couplings and the large geometry changes, as the energy director flows from its initial triangular shape till becoming a thin film at the interface. All couplings are handled using an iterative method that ensures a rigorous solution at convergence. Large geometry changes are treated within an Eulerian framework where the interface is described precisely using a level-set method. Due to computation time limitations, the problems presented 


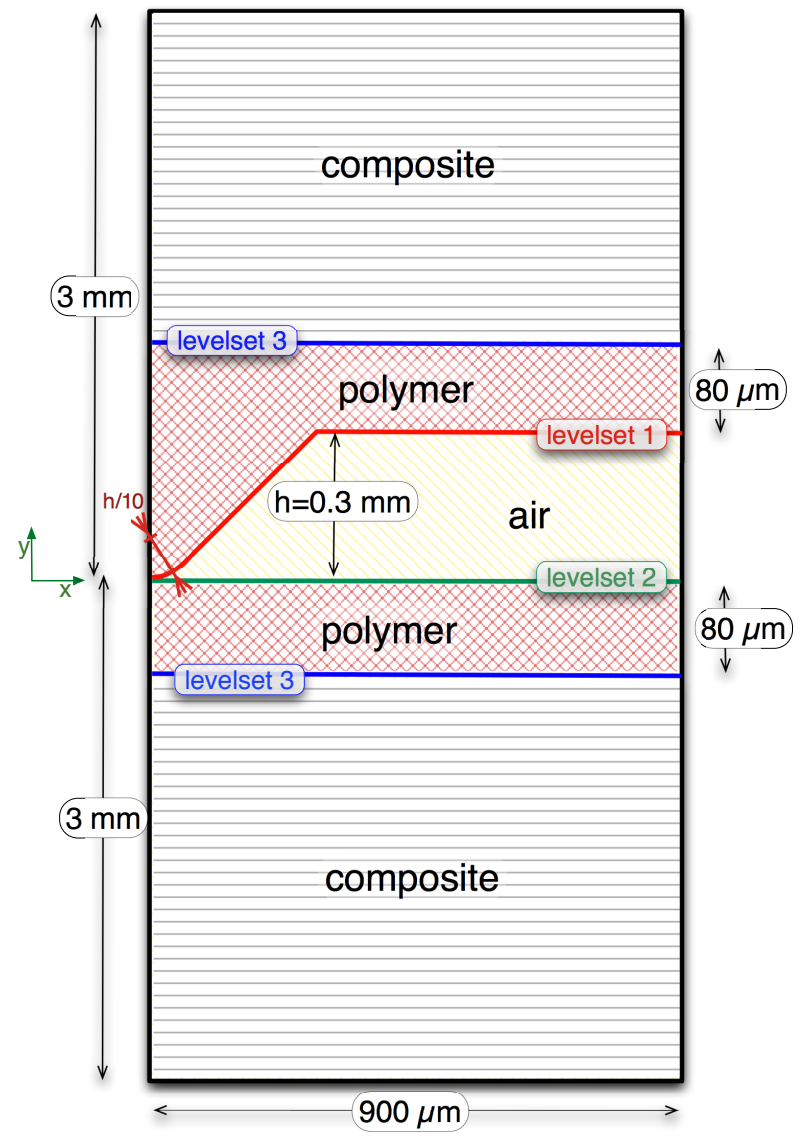

Fig. 3: Initial domain and materials used in the simulations.

in the following are solved on a two-dimensional domain in a plane strain assumption. We therefore consider the so-called "static" ultrasonic process, where the sonotrode does not move. The three-dimensional simulations still require numerical improvements that will be addressed in further works.

\subsubsection{Simulation cases}

Thanks to symmetries, the resolution domains is restricted to one half of an energy director and to one half step between two directors, as shown in Fig. 3.

The height of the domain allows one to consider the whole upper and lower composite plates. Fig. 3 shows that three level-set fields describe the geometry. The first one describes the energy director/air interface, the second one the lower plate/air interface, and the third one the composite/polymer interface. 
(a)

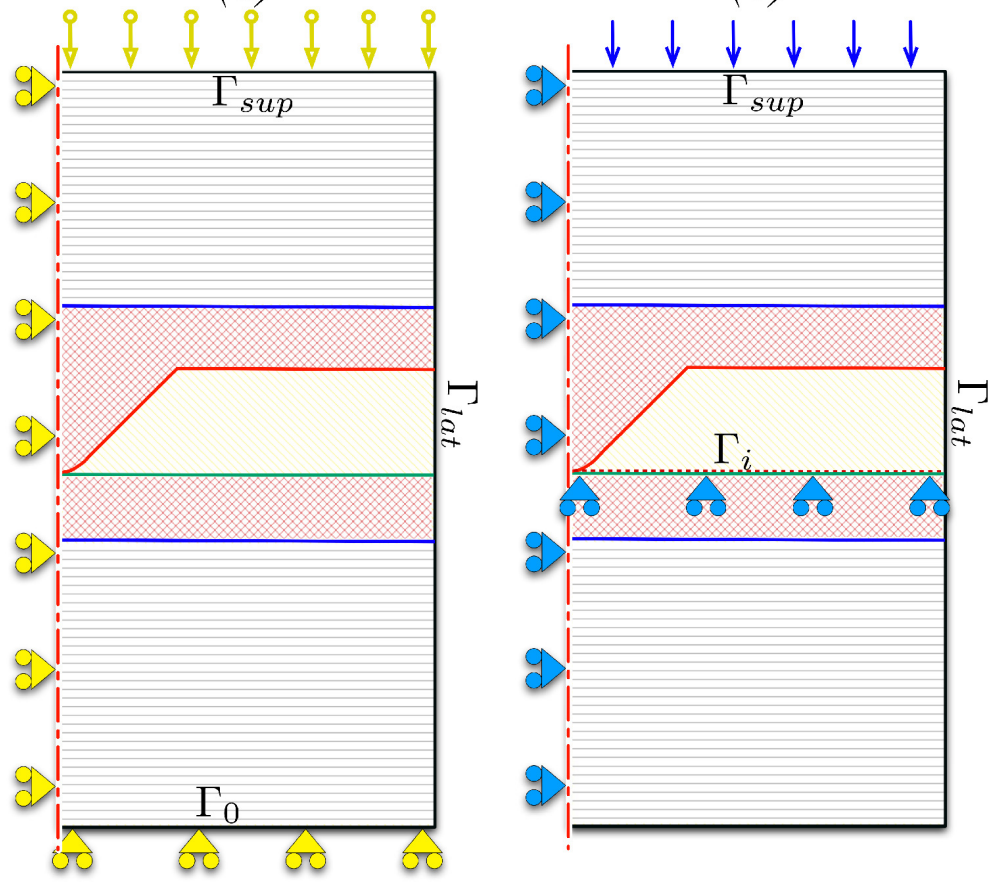

Fig. 4: Boundary conditions for the two mechanical problems: (a) elasticity, (b) fluid mechanics.

Note that, according to the industrial process, both composite plates have a superficial layer of neat polymer.

Boundary conditions for the mechanical problems are illustrated on Fig. 4. A symmetry condition is imposed on the left boundary. The vertical displacement of the lower part is imposed to zero in the elastic problem. For the fluid flow problem, the vertical velocity is imposed to zero on the internal boundary corresponding to the upper part of the lower plate. This allows one to simplify the handling of contact between the energy director and the lower plate. The lower plate is therefore considered as rigid in the flow problem.

The right boundary is let free in both the elastic and fluid problems, which enables to overcome the difficulty of extracting the air. Notice that this is closer to the condition encountered in the "continuous" process where the extraction of air is possible along the third dimension. As visible from table 1, it is also worth noting that the air mechanical properties are very low compared to the polymer, so that it will not affect the flow simulations.

On the upper boundary, a vertical displacement, corresponding to the amplitude of the sonotrode vibration, is imposed in the elastic problem. A vertical homogeneous stress distribution, corresponding to the holding force of the sonotrode, is imposed in the fluid mechanics problem. 
In the thermal problem, all boundaries are assumed perfectly insulated. Due to the short processing time and the rather low temperatures, thermal exchanges with the external medium do not have to be considered here. Doing so, the temperature raise in the system will be due only to the mechanical dissipation.

\subsection{Quality of welds}

First results obtained by Levy et al [13] show that the thermomechanical and topological evolution of energy directors is very complex so that it may be difficult to compare the efficiency of different processing conditions or different geometries. Probably due to the semi-crystalline nature of PEEK, the process can be decomposed into two rather well separated steps: (i) the initial heating due to the tip effect and (ii) the flow stage due to the softening of the polymer. It is often non trivial to decide whether one evolution is better than the other one so that some objective criteria are required. In particular, it is at least important to predict the degree of adhesion one can expect from a given set of processing parameters.

\subsubsection{Contact}

The first requirement for adhesion is obviously the presence of a contact at a microscopic scale, called intimate contact. It mainly depends on the surface roughness, the pressure between the two plates and the polymer behavior, as discussed in Lee and Springer [12] or Mantell and Springer [16].

In our case, the mesoscopic scale retained will be considered as small enough to determine whether the intimate contact is achieved or not. The adhesion process is therefore assumed to start when the two surfaces describing the energy director flow and the lower plate surface get in contact.

\subsubsection{Healing}

Once this intimate contact is achieved, the resulting interface can disappear in time. This is physically realized by diffusion of the polymer macromolecules across the interface [12] which can be modeled by the reptation theory [7]. In order to define the quality of the healing, many author define the healing degree $D_{h}$ as the ratio between the instantaneous interfacial bond strength and the ultimate interfacial bond strength $[12,20,5]$. Those authors propose to model this healing degree evolution using the so-called welding time $t_{w}$, for which a full interface bond strength is obtained :

$$
\frac{\partial D_{h}}{\partial t}=\left(\frac{1}{t_{w}(T)}\right)^{\frac{1}{4}} .
$$

Note that for low molecular weight polymers, this welding time is similar to the reptation time; but that for high molecular weight, it might be smaller 
(full strength is obtained before a full reptation of the macromolecule) [26]. The welding time dependency with temperature $T$ is classically modeled using the Arrhenius law:

$$
t_{w}=A_{r e p} \exp \left(\frac{E_{a}}{R T}\right)
$$

$A_{\text {rep }}$ being a pre-exponential coefficient depending on the material.

As suggested by Lee and Springer [12] or Cho and Kardos [6], the crystallinity of the matrix affects the accuracy of equation 5. Indeed, the crystal lamella in the semi-crystalline PEEK below melting temperature act as obstacles to the reptation and healing. Nonetheless this model has been widely used to model auto-adhesion in forming processes of semi-crystalline PEEK matrix composite $[12,16,20,5,1,2,26,11,23,10]$. In the following, we will present degrees of healing computed using the equation :

$$
D_{h}=\int_{t_{0}}^{t} \frac{1}{t_{w}(T)^{\frac{1}{4}}} d t .
$$

Nevertheless, we have to keep in mind that this value is well overestimated since crystallization is likely to limit healing. To account for this limitation by crystallization, a full multiphysical model predicting the melting and crystallization phenomena should be performed.

In the simulations presented in next sections, material parameters of equation (6) for the welding time are given in table 1 . They were deduced from rheological data provided in [17], and $t_{w}$ was identified as the longest relaxation time.

\section{Results and discussion}

The numerical tool used was already qualitatively validated by comparing simulation with experimental order of magnitude of the process [13]. It is here used to understand the physical mechanisms of ultrasonic welding as well as the influence of the main processing parameters.

\subsection{Reference simulation}

In this first section, a simulation is performed using material parameters given in table 1 , as close as possible to experimental data. Besides its usefulness as a reference when studying the influence of the process parameter, this simulation allows one to understand the physical phenomena that occur during the process. 
Table 1: Material parameters used in the reference simulation.

\begin{tabular}{lccc}
\hline & Polymer & Air & Composite \\
\hline Young's modulus $E(\mathrm{~Pa})$ & eq. $(1)$ & $5 \times 10^{3}$ & $9.3 \times 10^{9}$ \\
Poisson coefficient $\nu$ & 0.4 & 0 & 0.2 \\
Newtonian viscosity $A\left(\mathrm{~Pa} . \mathrm{s}^{\mathrm{m}}\right)$ & $5.6 \times 10^{-3}$ & $2 \times 10^{-5}$ & 10 \\
activation energy $E_{a}(\mathrm{~J})$ & $7.44 \times 10^{4}$ & 0 & $7.44 \times 10^{4}$ \\
Carreau time $\lambda(\mathrm{s})$ & 1 & 0 & 1 \\
Carreau power $m$ & 0.54 & 1 & 1 \\
conductivity $k\left(\mathrm{Wm}^{-1} \mathrm{~K}^{-1}\right)$ & 0.24 & $2.6 \times 10^{-2}$ & 0.72 \\
heat capacity $\rho c\left(\mathrm{Jm}^{-3} \mathrm{~K}^{-1}\right)$ & $1.3 \times 10^{6}+4500 T\left[{ }^{\circ} \mathrm{C}\right]$ & $10^{3}$ & $2.22 \times 10^{6}$ \\
loss modulus $E^{\prime \prime}(\mathrm{Pa})$ & $2010^{6}$ & 0 & 0 \\
\hline sonotrode pulsation $\omega\left(\mathrm{rad} . \mathrm{s}^{-1}\right)$ & $1.25 \times 10^{5}$ & - & - \\
healing coefficient $A_{r e p}(\mathrm{~s})$ & $7.3 \times 10^{-6}$ & - & - \\
\hline
\end{tabular}

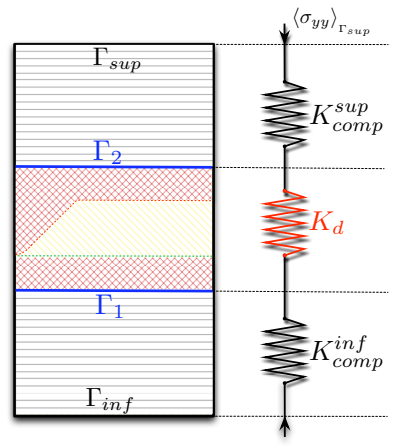

(a) Definition of equivalent stiffnesses of the different parts of the system.

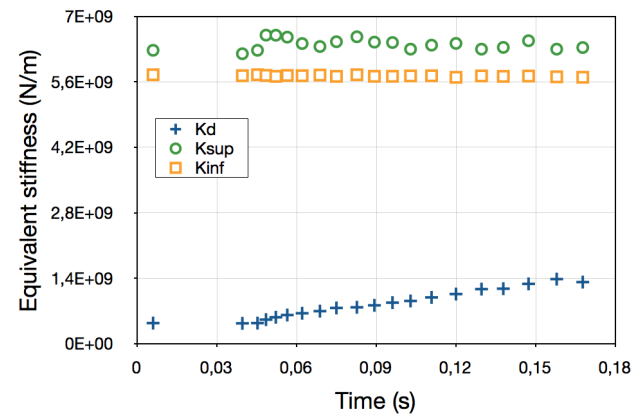

(b) Evolution of the equivalent stiffnesses during welding.

Fig. 5: Analysis of energy localization in the process.

\subsubsection{Phase I: heating}

During the initial phase the vibration strain is concentrated at the tip of the energy director. In order to quantify this localization, we consider the equivalent stiffness of three subdomains of the system, as shown on Fig. 5(a).

The stiffness of each zone is computed as the ratio between the vertical stress average and the average displacement difference between the two horizontal interfaces (successively $\Gamma_{\text {sup }}, \Gamma_{2}, \Gamma_{1}$ and $\Gamma_{\text {inf }}$ ) bounding each subdomain. Fig. $5(b)$ shows that the equivalent stiffness $K_{d}$ of the central part, containing the director is almost five times lower than the stiffness of the plates $K_{\text {sup }}$ and $K_{\text {inf }}$

This is explained by the triangular geometry of the director. It induces a strain concentration that produces a localization of the thermal dissipation (2) which leads to a local heating of the tip of the director, as shown on Fig. 6. 


114
104
95.4
86.4
77.3
68.3
59.2
50.1
41.1
32
23
TEMPERATURE

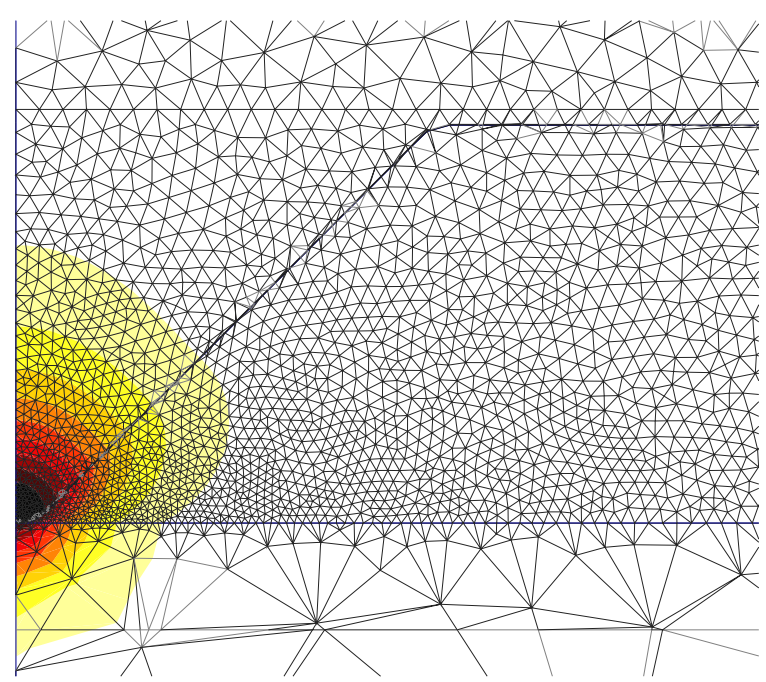

Fig. 6: Temperature field in the initial phase at time $t=0.03 \mathrm{~s}$ for the reference simulation case.

This is the so-called tip effect which can be quantified with the proposed equivalent stiffness estimator. One may think that the heating softens the material and improves the localization and the tip effect. But once the polymer softens, it begins to flow and the sharp geometry is rapidly lost. Therefore the equivalent stiffness of the director slightly therefore in time, as shown on Fig. $5(b)$. Due to the slow heat diffusion, the temperature then remains located around the energy director, which stiffness is 4 to 5 times smaller than that of the composites plates. In such a case, typical of current industrial conditions, one can see that the objective of bringing the energy directly at the interface is reached.

\subsubsection{Phase II: flow}

The local heating at the tip of the director softens the polymer which, in the second phase, flows at the interface. The highly localized tip effect initiates a special flow morphology which is referred to as fold morphology (cf. Fig. 7). It has already been highlighted and successfully compared to experimental morphology in previous work [13].

Fig. 8 represents the squeezing ratio evolution which is the displacement of the upper plate normalized by the energy director's initial height. It clearly illustrates the transition between the first stage (before $t=0.04 \mathrm{~s}$ ) where the tip effect occurs with almost no shape modifications and the second stage of flow and squeezing. In that second phase the squeezing speed progressively lowers as the fold fills the gap between the plates.

Note that the simulation does not account for crystallization phenomena. Nonetheless, it will influence the flow as mechanical properties are evolving 

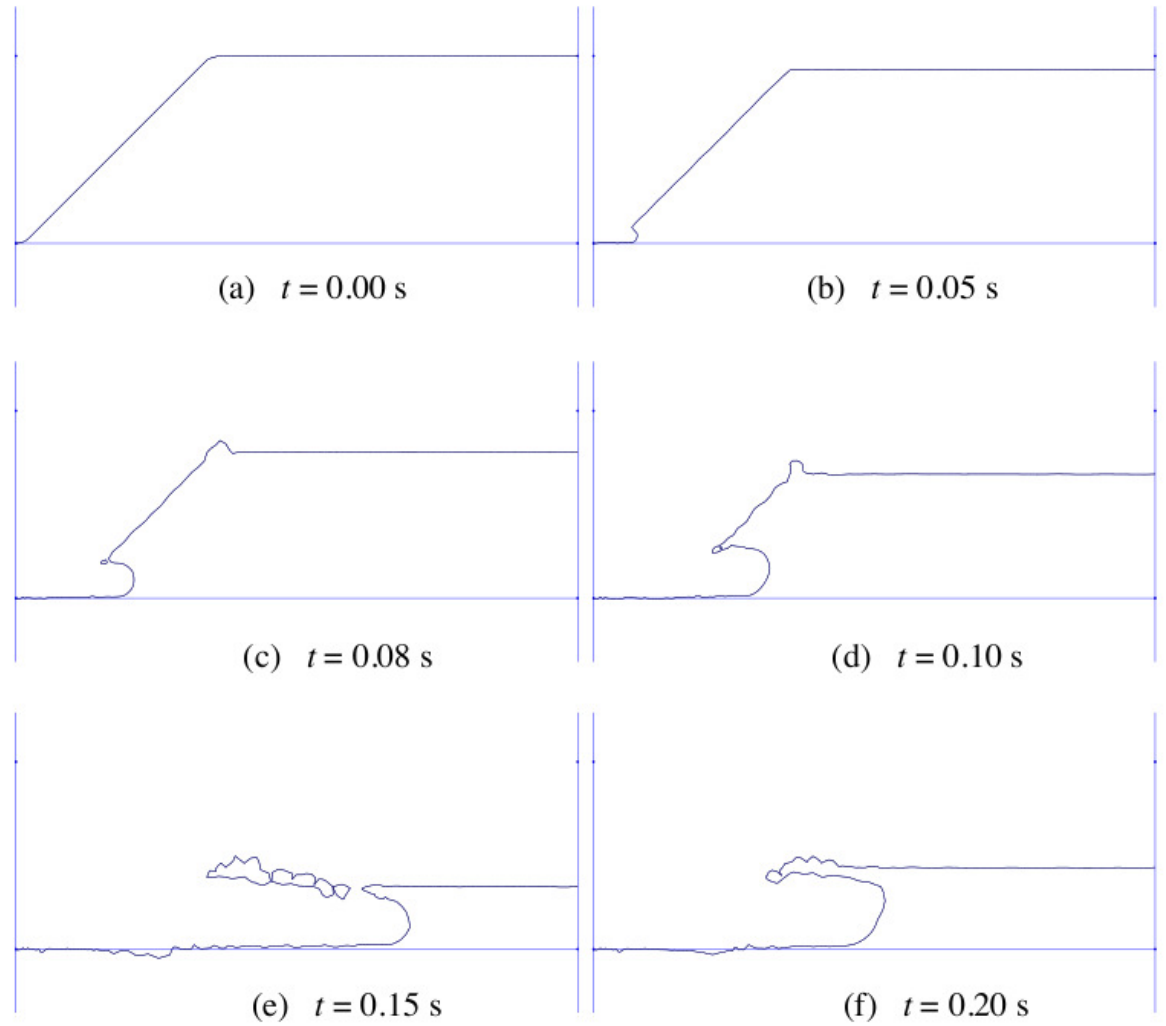

Fig. 7: Free surface evolution for the reference simulation: initiation and expansion of the fold.

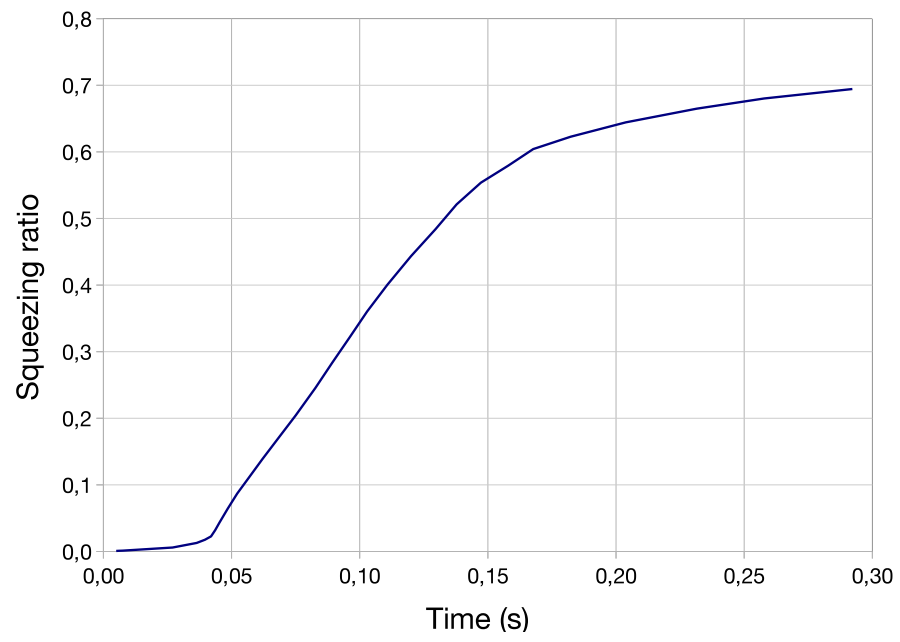

Fig. 8: Squeezing ratio vs. time. 


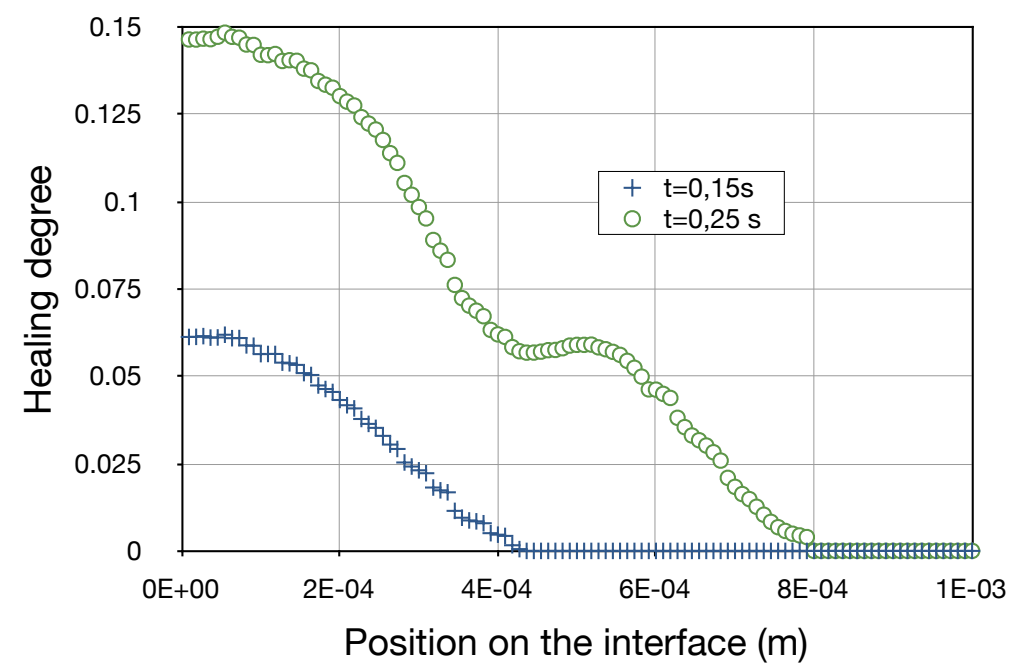

Fig. 9: Healing degree profiles along the welded interface at time $t=0.15 \mathrm{~s}$ and $t=0.25 \mathrm{~s}$

with the degree of crystallization. Therefore the presented results will loose accuracy when the temperature gets colder and crystallization occurs.

\subsubsection{Contact and healing}

The observed fold morphology (see Fig. 7) provides suitable conditions for adhesion. The hot flow front moves along the lower plate surface and achieves intimate contact, at least at the mesoscopic scale of the study. One can remark that a progressive (continuous) evolution of the fold along the lower plate is desired to ensure intimate contact and avoid air entrapment.

Thanks to this evolution, the local heating at the vicinity of the interface is ensured by the convection of the hot polymer, heated by the tip effect. To complete this analysis, Fig. 9 presents the healing degree as defined in section 2.2.2, computed along the interface for different times. As highlighted in section 2.2.2, the computed degree of healing is to be considered as a qualitative measurement because it does not account for crystallinity. Nonetheless, it shows the adequacy of the mesoscopic approach to study adhesion.

At the end of the flow phase, around $t=0.25 \mathrm{~s}, D_{h}$ is far from reaching 1 so that the interface would still not be healed at this time. This may be actually the case, but should be confirmed by more precise simulations including local adaptative mesh refinement and better material properties identification. Furthermore, the rapid evolution of the healing degree between $t=0.15 \mathrm{~s}$ and $t=0.25 \mathrm{~s}$ reasonably lets imagine that a good healing can be achieved during the following cooling phase. In order to predict the final quality of the adhesion a study of the following cooling phase is required. 
Finally, at this stage, more than a quantitative estimator of the adhesion, the healing degree allows one to estimate and predict the homogeneity of the adhesion at the interface. For instance, the healing degree profile on Fig. 9 for time $t=0.25 \mathrm{~s}$ exhibits a zone of lower healing degree. This is caused by the fold evolution that has not been sticking along the lower plate during the whole flow. Intimate contact was lost for few instants.

The analyses of the heating and flow phases is possible thanks to our novel simulation tool that handles simultaneously both phenomena. In the next sections, we propose a parametric analysis and discuss the influence of some process parameters on those two interdependent stages of the mesoscopic process.

\subsection{Parametric analysis}

In this section, the influences of some process parameters on the heating and flow of polymer are studied. Since the numerical tool is qualitative, results will only highlight trends and do not pretend to predict accurate quantitative values.

\subsubsection{Amplitude of vibration of the sonotrode}

The dissipation term (2) is proportional to the square of the elastic strain. The process is therefore highly dependent on the amplitude of vibration imposed on the upper boundary by the sonotrode. The initial maximum heating speed at the tip of the director is represented versus imposed amplitude on Fig. 10. As expected, the quadratic effect is exactly recovered for this heating phase.

One has to notice that the sonotrode does not impose a strictly sinusoidal displacement to the upper plate. In practice, due to inertia effects, the contact may be lost causing hammering as explained by Nonhof and Luiten [18]. Hence, increasing vibration amplitude of the sonotrode may lengthen the loss of contact with the upper plate. The quadratic dependence observed in our mesoscopic simulation may therefore not be observed in practice.

\subsubsection{Holding force of the sonotrode}

Besides the vibration imposed by the sonotrode, the contact time between the sonotrode and the upper plate is highly dependent on the holding force of the sonotrode, as shown by Ha Minh [9]. Hereunder, we do not consider such macroscopic effects and focus on the effect of the holding force on the mesoscopic flow at the interface, under the assumption of a permanent contact between the sonotrode and the upper plate.

Fig. 11 shows that when increasing the holding force, the maximal temperature at the tip of the director is lowered. Indeed, imposing a higher holding 


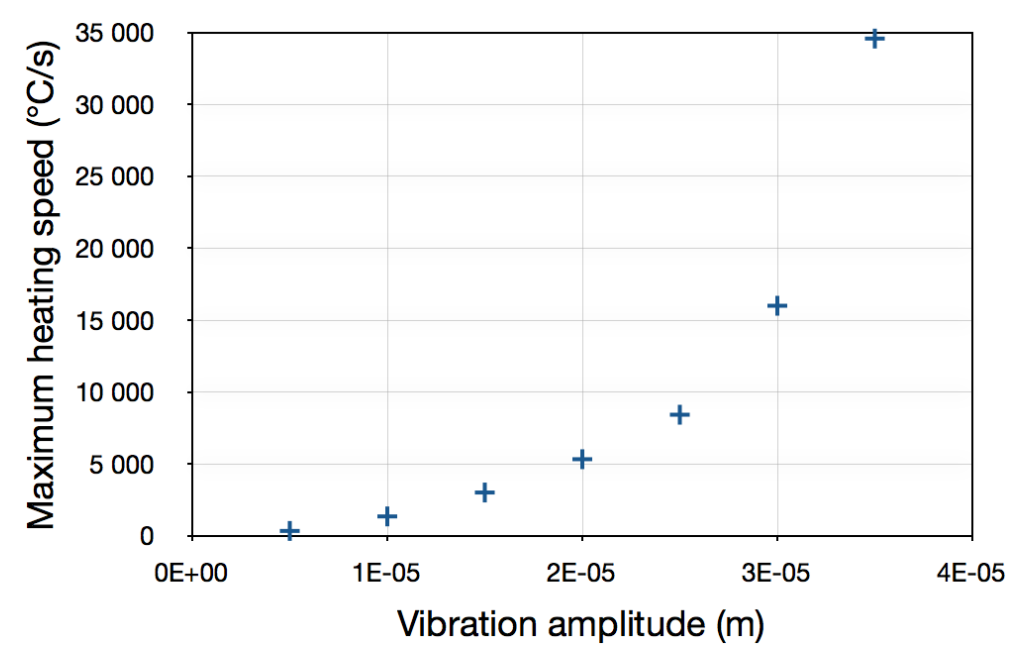

Fig. 10: Maximum initial heating speed in the early stages of squeezing versus amplitude of vibrations imposed on the upper boundary.

force results in a faster squeezing of the director. The flow, which occurs earlier, limits the tip effect and prevents the polymer from reaching a high temperature. Surprisingly, a lower holding force of the sonotrode allows a better heating.

The holding force of the sonotrode therefore has two antagonistic effects, which require to find an optimum:

1. A high holding force value results in a longer contact time between the tool and the upper plate, and therefore, a better energy transfer and higher dissipation.

2. A lower holding force value results in a delay in the flow, a longer heating phase and higher temperature reached.

\subsubsection{Thickness and stiffness of the plates}

In section 3.1.1, the equivalent stiffness of the plates were estimated about 5 times greater than that of the director. If the stiffness of the plates is lowered by a factor of 5 or more, it reaches the equivalent stiffness of the energy director, that will no more play the role of an energy concentrator. This would be the case if the material of the plates was softer or if plates were thicker. Fig. 12 shows that for softer plates (which Young modulus was lowered from $E_{\text {plates }}=9.3 \mathrm{GPa}$ to $\left.E_{\text {plates }}=2 \mathrm{GPa}\right)$, the temperature does not increase enough to initiate the flow. This result is confirmed by the experience. It is known to be difficult or even impossible to weld thick plates using such a process. 


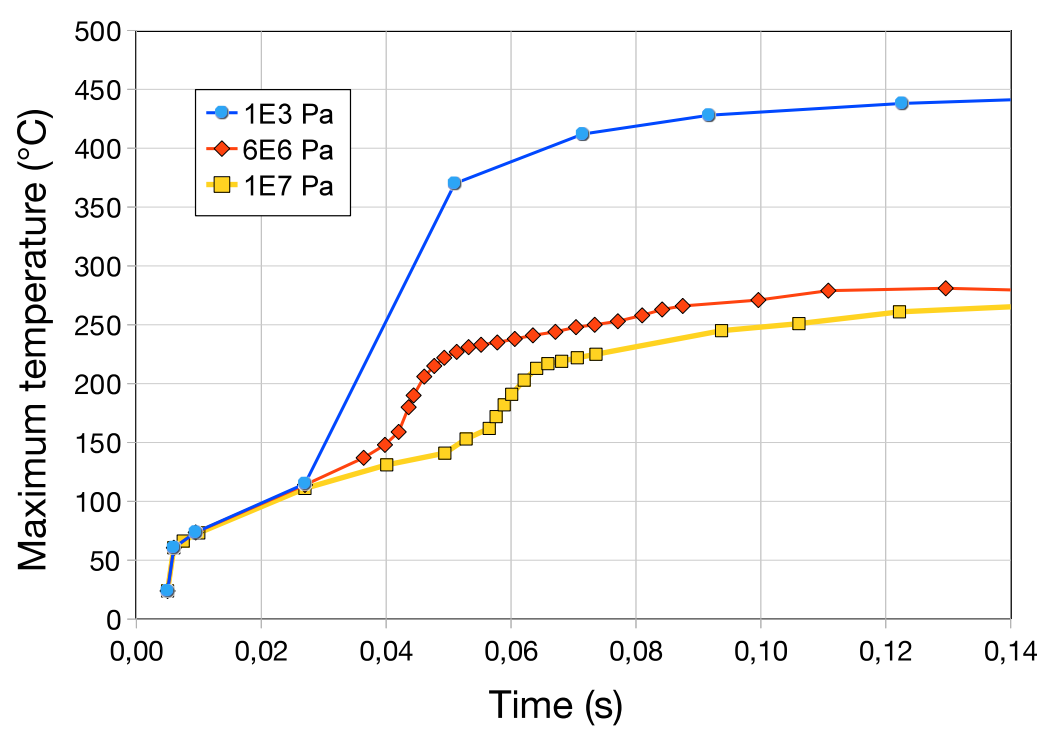

Fig. 11: Maximal temperature in the director versus time for different holding forces of the sonotrode, imposed as a uniform normal stress with equivalent resulting force.

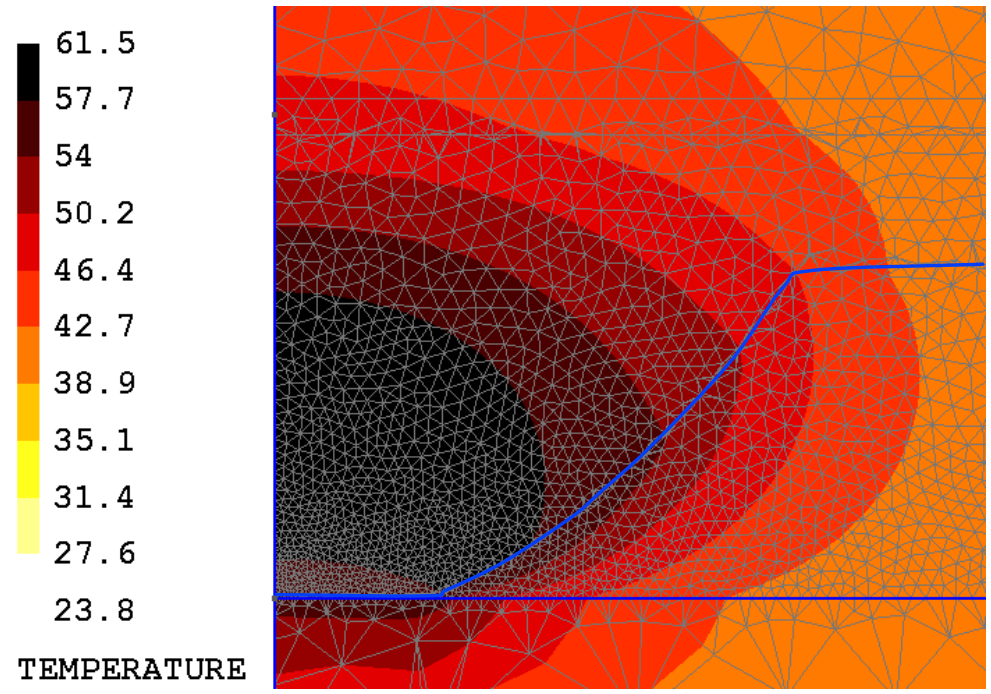

Fig. 12: Temperature field at time $t=5 \mathrm{~s}$ for soft plates where $E_{\text {plates }}=2 \mathrm{GPa}$ and for the same holding force as in the reference simulation. 


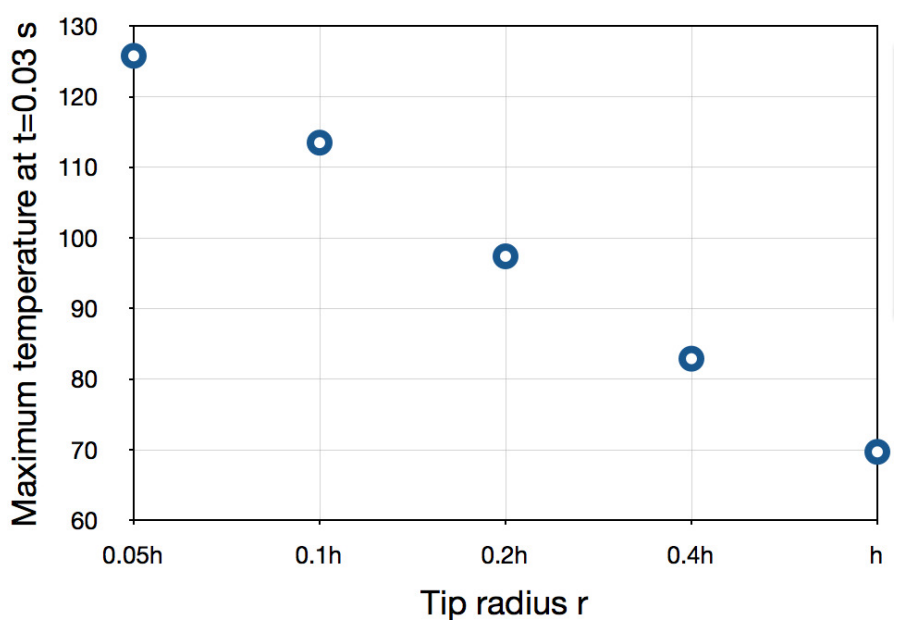

Fig. 13: Maximum temperature in the director at time $t=0.03 \mathrm{~s}$ versus radius of curvature $r$ at the tip of the director.

\subsubsection{Radius of curvature at the director's tip}

In the reference simulation, the radius of curvature $r$ at the tip of the director is $10 \%$ of the director's height $h$, according to the real geometry. Simulations with different radii of curvature were also performed. Fig. 13 represents the maximum temperature at the director tip during the initial heating phase, versus the radius of curvature $r$. This temperature decreases somewhat linearly while increasing $r$. As expected, sharper energy directors result in a faster heating during the initial phase.

In order to illustrate the tip effect on the flowing phase, Fig. 14 presents a comparison of the resulting flow fronts for two different radii. As the flow is not initiated as quickly in both configurations, plots are given for different instants were the fold morphology is comparable. In addition, the difference between both temperature fields is also given. Fig. 14 thus shows that the temperature field and deformed shape for $r=0.05 h$ is very close to the one for $r=0.2 h$ taken a bit earlier. Indeed, the relative difference

$$
\frac{T(r=0.05 h, t=0.065 \mathrm{~s})-T(r=0.2 h, t=0.09 \mathrm{~s})}{T(r=0.2 h, t=0.065 \mathrm{~s})}
$$

is small and never exceeds 0.1 . A smoother director therefore only leads to a delay in the flow.

\subsubsection{Angle of the triangular director}

In this last section, simulations obtained with different angles of the director are analyzed. The different simulations were performed using equal amounts 


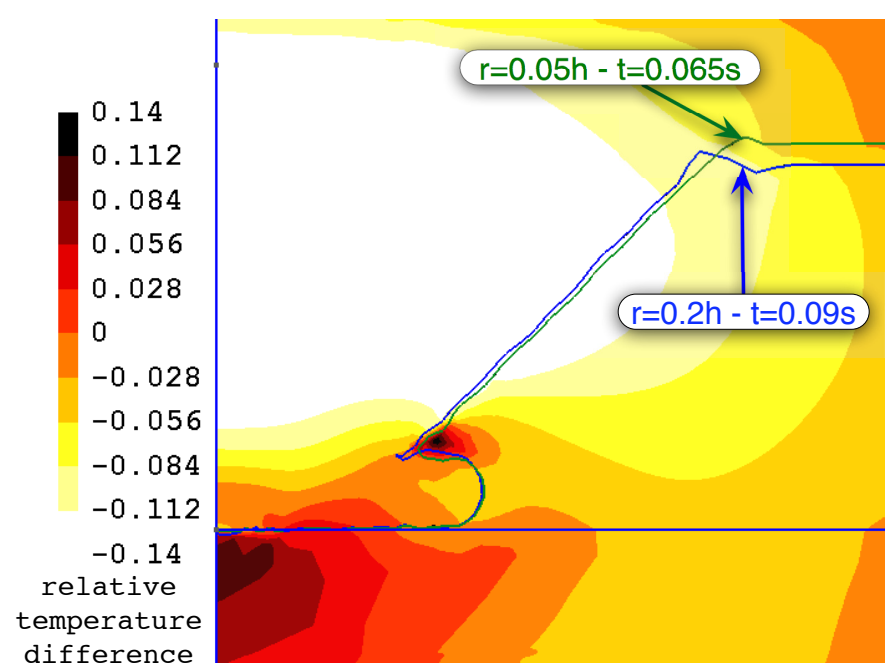

Fig. 14: Deformed shapes and relative temperature difference between: - an initial tip radius $r=0.05 h$ at time $t=0.065 \mathrm{~s}$ and - an initial tip radius $r=0.2 h$ at time $t=0.09 \mathrm{~s}$.

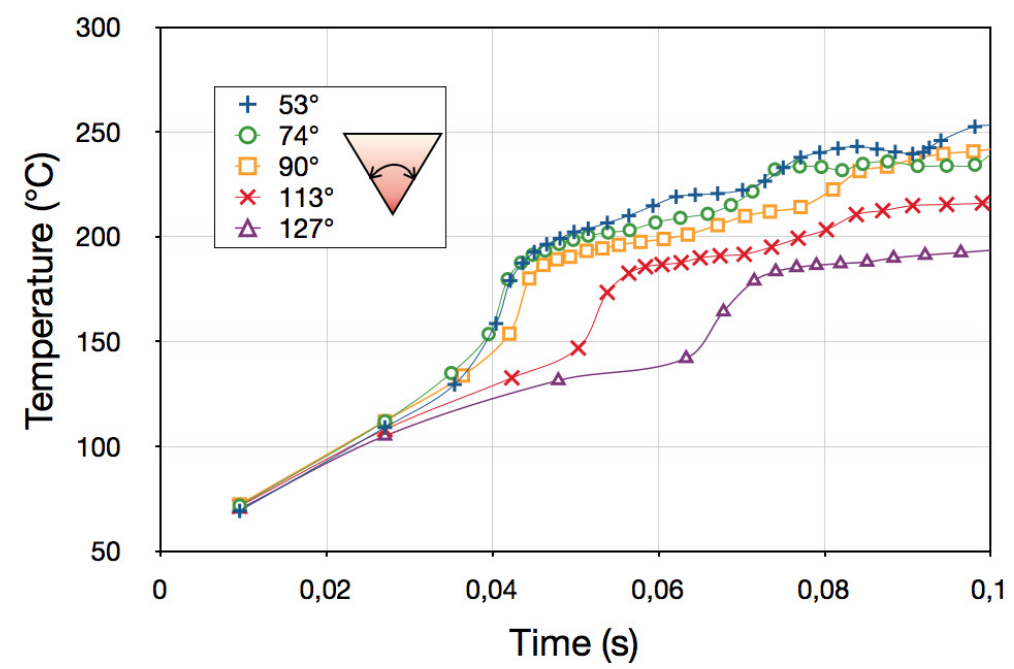

Fig. 15: Maximum temperature at the tip of the director versus time for different angles of directors.

of polymer (which means different height of director) and equal radius of curvature at the tip.

Fig. 15 represents the temperature at the tip of the director versus time for different angles. Since the radius of curvature is identical, during the initial phase, the increase of temperature does not depend on the angle. During the 


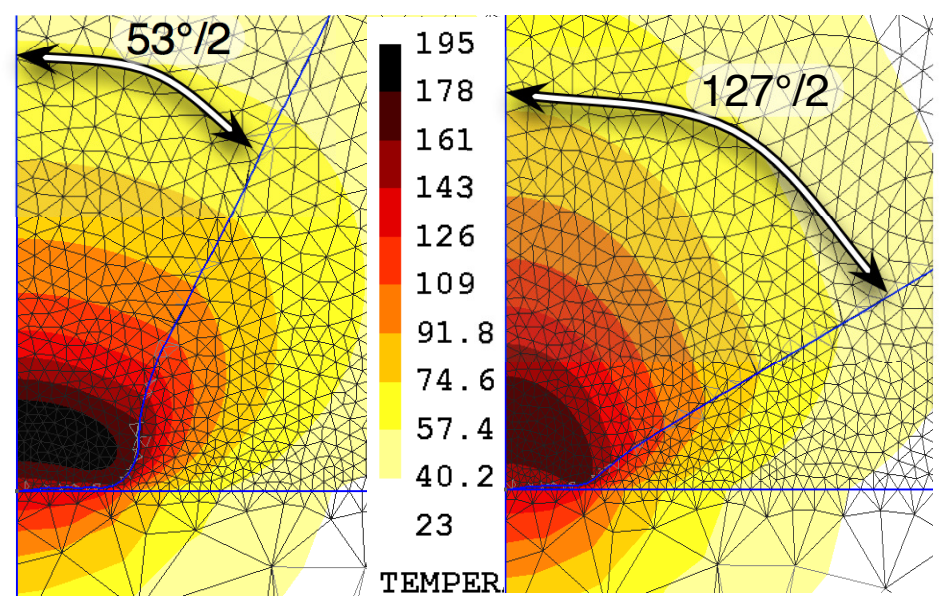

Fig. 16: Temperature fields for equivalent macroscopic squeezing ratios. Initial angle of $53^{\circ}$ at time $t=0.035 \mathrm{~s}$ and $127^{\circ}$ at time $t=0.05 \mathrm{~s}$.

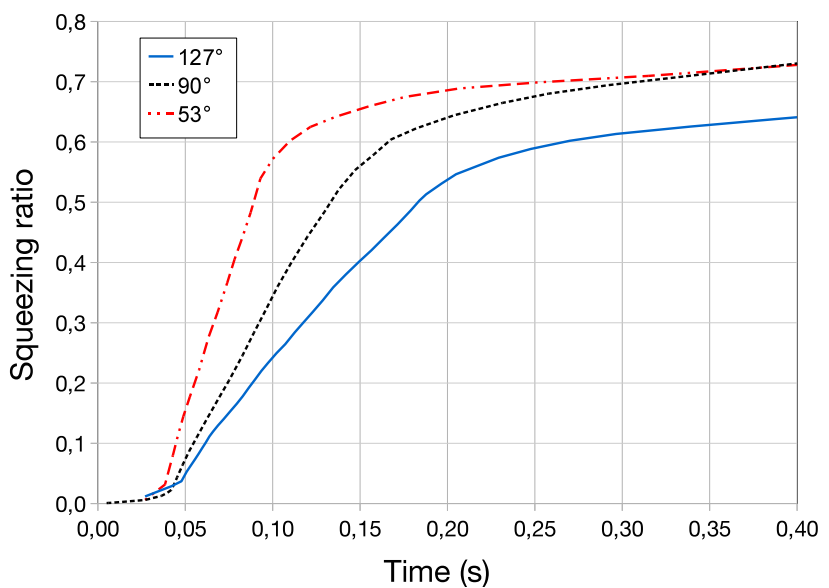

Fig. 17: Squeezing ratio versus time for different initial angle of director.

flow phase, the temperature stabilizes (final times on the graph). Moreover, the transition appears later for larger angles. This is explained by the better thermal insulation of a sharper geometry which limits the diffusion of heat in the director. The temperature is indeed more localized and higher, as shown on Fig. 16. If we now look at the squeezing time, as presented on Fig. 17, it is clear that sharper directors enable faster processing conditions.

However, considering Fig. 15 again, we observe a sharper transition regime for small angles. This is associated with the moment when the fold reaches the height of the gap, leading to a stress transfer over a much wider zone. As 


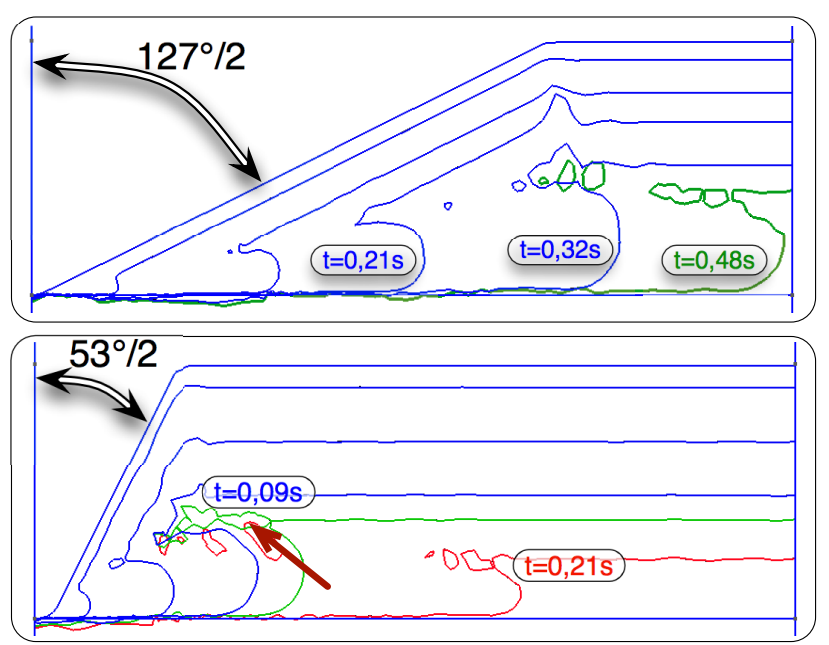

Fig. 18: Flow morphology for two extreme initial angles of energy directors.

illustrated by Fig. 18, the drawback of sharp energy directors, due to the more pronounced fold, is that they will lead to more air entrapment, indicated by an arrow on the figure.

Those simulation clearly show that non evident conclusion can be drawn regarding the geometry of energy directors. Results however show that an optimum shape could be found using a more complex geometry, with first a rather sharp end in order to better concentrate the heating, followed by a progressive enlargement, that would ensure better contact conditions.

\section{Conclusion}

In this paper, we presented the application of an original simulation tool to the physical analysis of the ultrasonic welding process. The two main strengths of this code, already presented in a previous paper [13], consist in the use of (i) a level set technique within an Eulerian framework that enables to handle the large geometry changes with a precise description of the physical surfaces, and of (ii) an iterative multiphysical solver that allows one to solve rigorously two mechanical problems and a thermal problem. At this stage, this new code is still a demonstrator but is not optimized for large computations. It is still not applicable to the simulation of the full 3D continuous process involving an advance of the sonotrode along the energy director (the out of plane direction in our $2 \mathrm{D}$ representation).

However, much of the results presented here in the $2 \mathrm{D}$ case can be extended to $3 \mathrm{D}$ situations. For instance, the fact that a high sonotrode holding force will squeeze the director can be extended to the continuous process, the difficulty to weld thick plates was also already observed experimentally. On the other 
hand the results concerning the influence of the director angle on the flow morphology can not be extended directly to the continuous welding process. Nonetheless they provide some expectations. Finaly, the $2 \mathrm{D}$ results give a better understanding of the complex and non evident interactions between geometry, process parameters, thermics and mechanics.

A particular effort was made in this work to choose realistic material parameters and laws that can represent at best the semi-cristalline behavior of the PEEK. Thanks to those models, results show that one can distinguish two stages in the welding process: $(i)$ a stage of localized heating due to the tip effect until reaching a sufficient drop of mechanical properties followed by $(i i)$ a flow stage where a fold of polymer is formed and moves forward until filling the gap between the plates and eventually achieving adhesion. One may notice that the mesoscopic scale retained in this study is inescapable for analyzing those phenomena. The study must therefore be carried at this scale to physically determine the adhesion or the formation of porosity. We also remind that the results and especially the healing degrees presented above are obtained after the flow phase. The subsequent cooling phase, that was not studied in this work, is needed if one wants to fully predict the final quality of the weld.

In order to better understand the complex interactions between physical phenomena, the present work then focused on the analysis of several parameters on both stages of the process, trying to draw conclusions on the potential quality of the realized assembly. Several expected important technological results were first confirmed by this study:

- The amplitude of vibrations acts directly on the provided energy and governs the local heating rate. However, in practice, one would have to account for the hammering phenomenon that limits this effect and therefore reduces the overall efficiency of the process.

- The geometry of the tip of directors is essential to reach rapidly the melting temperature. One should use a tip radius of curvature as small as possible.

- The analysis in equivalent stiffness is a very useful and simple tool one can obtain by a simple elastic calculation. It enables to quantify the energy concentration at the interface, initial requirement for the efficiency of the whole process.

Two other important results which are less evident where also highlighted:

- Firstly, a too high holding force leads to a poor welding for it squeezes the energy director before it has reached a high temperature.

- Secondly, results regarding the geometry show two antagonist effects. A sharp director shape leads to a rapid and well localized heating, thus enabling the creation of a hot fold promoting a good adhesion. However, the final squeezing of the fold may entrap porosities and so weaken the realized join. On the contrary, a flat director shape, though slower for the heating stage, leads to a more progressive filling of the interface with less porosities.

The parametric analysis detailed in this paper therefore brings a deeper understanding of the ultrasonic welding process which is essential for any further 
optimization of processing parameters. Nevertheless, in order to accurately determine the final healing degree of the interface, some additional work is still needed. First, the final cooling phase is to be investigated. Then, the numerical tool has to be validated and maybe improved, in order to give more accurate results, especially concerning the healing degree evolution which depends on crystallization. These improvements may give quantitative results concerning the "static" process, where the sonotrode does not advance. The next phase is clearly to extend the analysis to a three dimensional domain, in order to simulate the continuous process with the advancing of the sonotrode.

Acknowledgements This study was supported by Eric Soccard and Mathieu Piana from EADS IW. We express our thanks for their knowledge about the technology of ultrasonic welding.

\section{References}

1. Ageorges C, Ye L, Mai YW, Hou M (1998) Characteristics of resistance welding of lap shear coupons : Part ii : Consolidation. Composites 29A:911-919

2. Ageorges C, Ye L, Hou M (2001) Avdances in fusion bonding techniques for joining thermoplastic matrix composites: a review. Composites Part A: applied science and manufacturing 32(6):839-857

3. Ahmed T, Stavrov D, Bersee H, Beukers A (2006) Induction welding of thermoplastic composites-an overview. Composites Part A: Applied Science and Manufacturing 37(10):1638-1651

4. Benatar A, Gutowski TG (1989) Ultrasonic welding of PEEK graphite APC-2 composites. Polymer Engineering and Science 29(23):1705

5. Butler C, Mccullough R, Pitchumani R, Gillespie Jr J (1998) An analysis of mechanisms governing fusion bonding of thermoplastic composites. Journal of Thermoplastic Composite Materials 11(4):338

6. Cho BR, Kardos JL (1995) Consolidation and self-bonding in poly(ether ether ketone) (PEEK). Journal of applied polymer science 56(11):14351454

7. De Gennes $\mathrm{P}$ (1971) Reptation of a polymer chain in the presence of fixed obstacles. The Journal of Chemical Physics 55:572

8. Goyal R, Tiwari AN, Mulik UP, Negi YS (2006) Dynamic mechanical properties of $\mathrm{Al}_{2} \mathrm{O}_{3} /$ poly(ether ether ketone) composites. Journal of Applied Polymer Science 104(1):568-575

9. Ha Minh D (2009) Couplages thermo-mécaniques lors de la soudure par ultrasons. application aux thermoplastiques. $\mathrm{PhD}$ thesis, Ecole Nationale des Ponts et Chaussées. ParisTech.

10. Khan MA, Mitschang P, Schledjewski R (2010) Identification of some optimal parameters to achieve higher laminate quality through tape placement process. Advances in Polymer Technology 29(2):98-111 
11. Lamethe JF, Beauchene P, Leger L (2005) Polymer dynamics applied to PEEK matrix composite welding. Aerospace Science and Technology $9(3): 233-240$

12. Lee W, Springer G (1987) A model of the manufacturing process of thermoplastic matrix composites. Journal of Composite Materials 21(11):1017

13. Levy A, Le Corre S, Chevaugeon N, Poitou A (2011) A levelset based approach for the finite element simulation of a forming process involving multiphysics coupling : ultrasonic welding of thermoplastic composites. European Journal of Mechanics - A/Solids 30(4):501-509

14. Levy A, Le Corre S, Poitou A, Soccard E (2011) Ultrasonic welding of thermoplastic composites, modeling of the process using time homogenization. International Journal for Multiscale Computational Engineering $9(1): 53-72$

15. Li R (1999) Time-temperature superposition method for glass transition temperature of plastic materials. Materials Science and Engineering A 278(1-2):36-45

16. Mantell S, Springer G (1992) Manufacturing process models for thermoplastic composites. Journal of Composite Materials 26(16):2348

17. Nicodeau C (2005) Modélisation du soudage en continu des composites a matrice thermoplastique. PhD thesis, Ecole Nationale Superieure d'Arts et Métiers de Paris, http://pastel.paristech.org/1506/

18. Nonhof C, Luiten G (1996) Estimates for process conditions during the ultrasonic welding of thermoplastics. Polymer Engineering and Science $36(9): 1177$

19. Soccard E (2007) Ultrasonic assembly method. Patent number: WO 2007/003626 A1 WO 2007/003626 A1, EADS CCR

20. Sonmez F, Hahn H (1997) Analysis of the on-line consolidation process in thermoplastic composite tape placement. Journal of Thermoplastic Composite Materials 10(6):543

21. Stavrov D, Bersee H (2005) Resistance welding of thermoplastic composites-an overview. Composites Part A: Applied Science and Manufacturing 36(1):39-54

22. Suresh K, Roopa Rani M, Prakasan K, Rudramoorthy R (2007) Modeling of temperature distribution in ultrasonic welding of thermoplastics for various joint designs. Journal of Materials Processing Technology 186(13):138-146

23. Tierney J, Gillespie JW (2006) Modeling of in situ strength development for the thermoplastic composite tow placement process. Journal of Composite Materials 40(16):1487-1506

24. Tolunay MN, Dawson PR, Wang KK (1983) Heating and bonding mechanisms in ultrasonic welding of thermoplastics. Polymer Engineering and Science 23(13):726-733

25. Wang X, Yan J, Li R, Yang S (2006) FEM investigation of the temperature field of energy director during ultrasonic welding of PEEK composites. Journal of Thermoplastic Composite Materials 19(5):593 
26. Yang F, Pitchumani R (2002) Healing of thermoplastic polymers at an interface under nonisothermal conditions. Macromolecules 35(8):3213-3224 